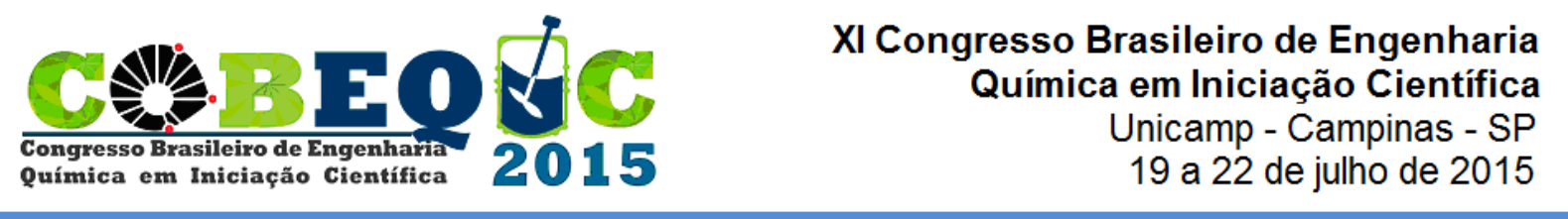

\title{
PLANILHAS EXCEL/VBA PARA PROBLEMAS ENVOLVENDO EQUILÍBRIO LÍQUIDO-VAPOR EM SISTEMAS BINÁRIOS
}

\author{
L. G. Oliveira, J. K. S. Negreiros, S. P. Nascimento, J. A. Cavalcante, N. A. Costa \\ Universidade Federal da Paraíba, Departamento de Engenharia Química \\ E-mail para contato: nagelalves@hotmail.com
}

\begin{abstract}
RESUMO - Este trabalho tem por finalidade fornecer uma planilha eletrônica com programação em objeto-modelo VBA para problemas envolvendo o equilíbrio líquido-vapor de sistemas binários. Para a fase líquida são considerados os sistemas ideal e não ideal, sendo esse corrigido através de um dos seguintes modelos: Margules 3 sufixos; van Laar; Wilson; NRTL e UNIQUAC. O trabalho é dividido em cinco partes: 1) criação do banco de dados contendo os parâmetros dos modelos de atividade; 2) criação de caixas de diálogos para interação do usuário com o programa; 3) codificações VBA para a determinação das condições adequadas do equilíbrio de fases; 4) transporte dos resultados do VBA para a planilha de entrada e saída de dados e 5) validação dos resultados fornecidos pelo programa. O resultado final é um aplicativo para fins educacionais e/ou comerciais, capaz de fornecer aos usuários as facilidades de um programa com aparência e estrutura de comandos consistentes com programa para Windows.
\end{abstract}

\section{INTRODUÇÃO}

A termodinâmica do equilíbrio de fases trata de estabelecer as relações entre as propriedades da regra das fases de Gibbs (temperatura, pressão e composição) que predominam quando duas ou mais fases alcançam o estado de equilíbrio. O equilíbrio entre fases é de interesse na Engenharia Química, uma vez que várias operações industriais consistem no contato entre duas ou mais fases. A regra das fases de Gibbs determina as condições necessárias para o equilíbrio, ou seja, permite estabelecer as características geométricas do equilíbrio de fases. A partir do critério de equilíbrio, modelos matemáticos são estabelecidos para descrever o comportamento das fases. Finalmente, rotinas computacionais são elaboradas para solucionar o modelo matemático estabelecido. Para os casos que não são permitidos hipóteses simplificadoras, os cálculos exigem esquemas iterativos em virtude da complexa dependência funcional entre as propriedades do modelo estabelecido.

\section{METODOLOGIA}

A metodologia empregada para o desenvolvimento da planilha Excel/VBA é apresentado na Figura 1. 
Figura 1 - Esquema do desenvolvimento do aplicativo

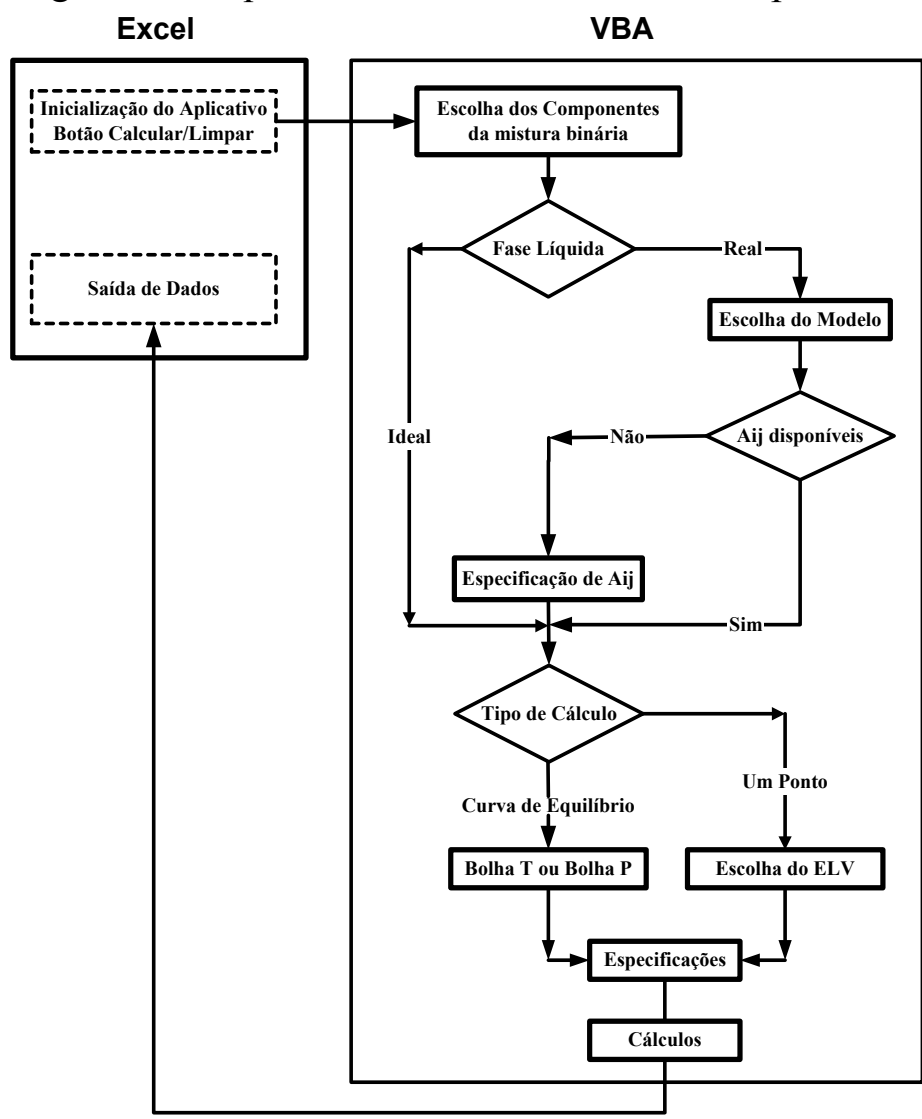

\subsection{Esquema do desenvolvimento do aplicativo}

Banco de dados: $\mathrm{O}$ desenvolvimento do aplicativo principia-se com a criação de dois bancos de dados. O primeiro contém uma lista de espécies químicas, constantes da equação de Antoine (Reid et al., 1977), volumes molares, parâmetros de áreas e de volumes moleculares (Gmehling et al., 1982a e 1982b). O segundo contém os parâmetros de interações binárias dos modelos de atividade de 200 sistemas comumente empregados na indústria petroquímica (Gmehling et al., 1982a e 1982b).

Escolha das Espécies Químicas: os componentes presentes na mistura binária são escolhidos através da caixa de diálogo mostrada na Figura 2.

Figura 2 - Seleção dos componentes da mistura

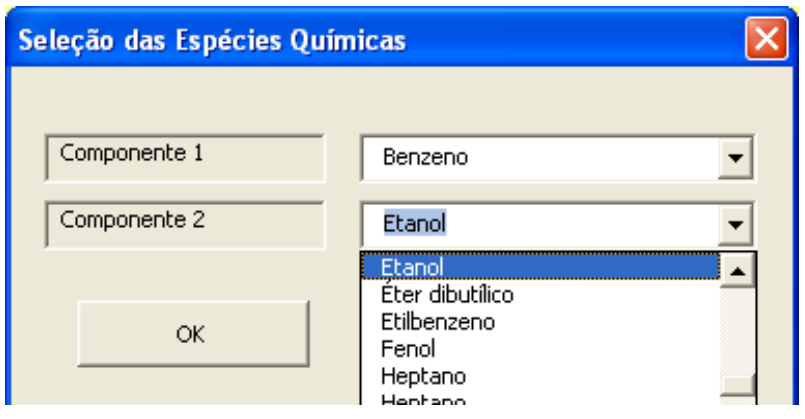


Se a escolha dos componentes estiver fora da ordem decrescente de volatilidade, o programa utiliza uma subrotina de ordenação por contagem proposta por Knuth (1999) para colocar os componentes na ordem decrescente de volatilidade.

Opções para a fase líquida: O usuário pode escolher entre sistemas ideais e reais na caixa de diálogo da Figura 3. Se a escolha for sistemas reais, a correção da fase líquida é realizada através da seleção de um modelo de atividade na caixa de diálogo da Figura 4.

Figura 3 - Seleção do tipo de fase líquida

Figura 4 - Seleção para a correção da fase líquida
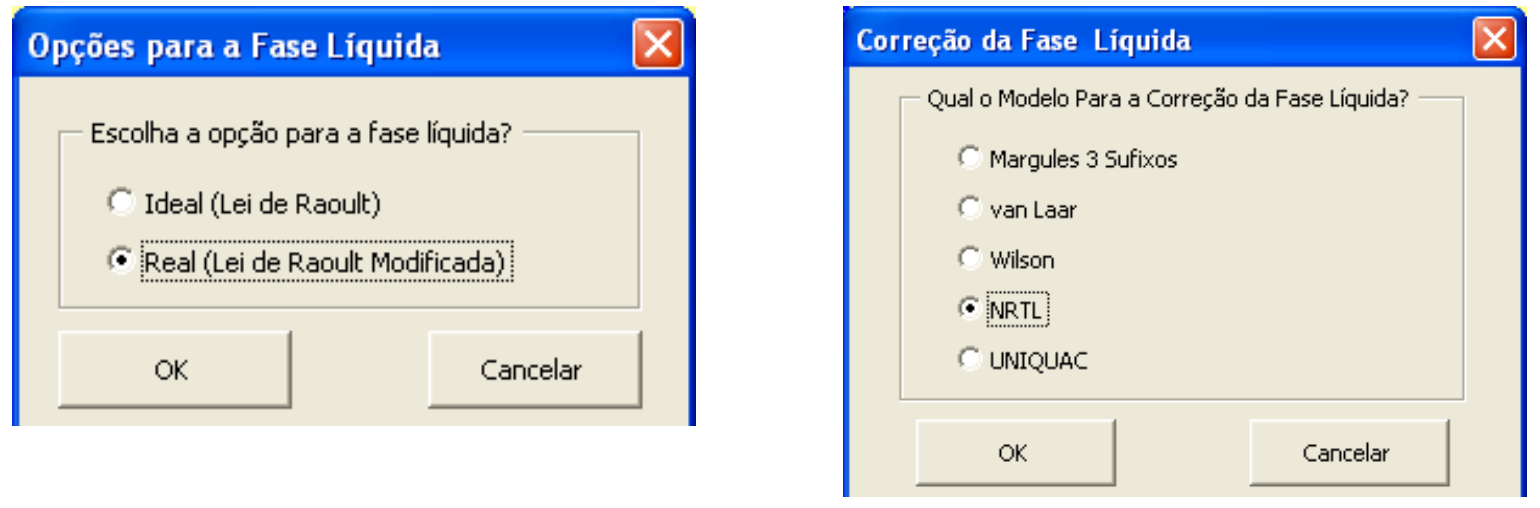

Se a opção escolhida for fase líquida ideal, os coeficientes de atividade têm valores iguais a unidade. Se a fase líquida escolhida for real, o aplicativo verifica a disponibilidade dos parâmetros binários no banco de dados usando a concatenação dos nomes dos componentes especificados na etapa anterior. Se o sistema é encontrado no banco de dados, os parâmetros binários são obtidos através da função de planilha Excel PROCV (VLOOKUP) e apresentados na Figura 5.

Figura 5 - Apresentação dos parâmetros obtidos no banco de dados

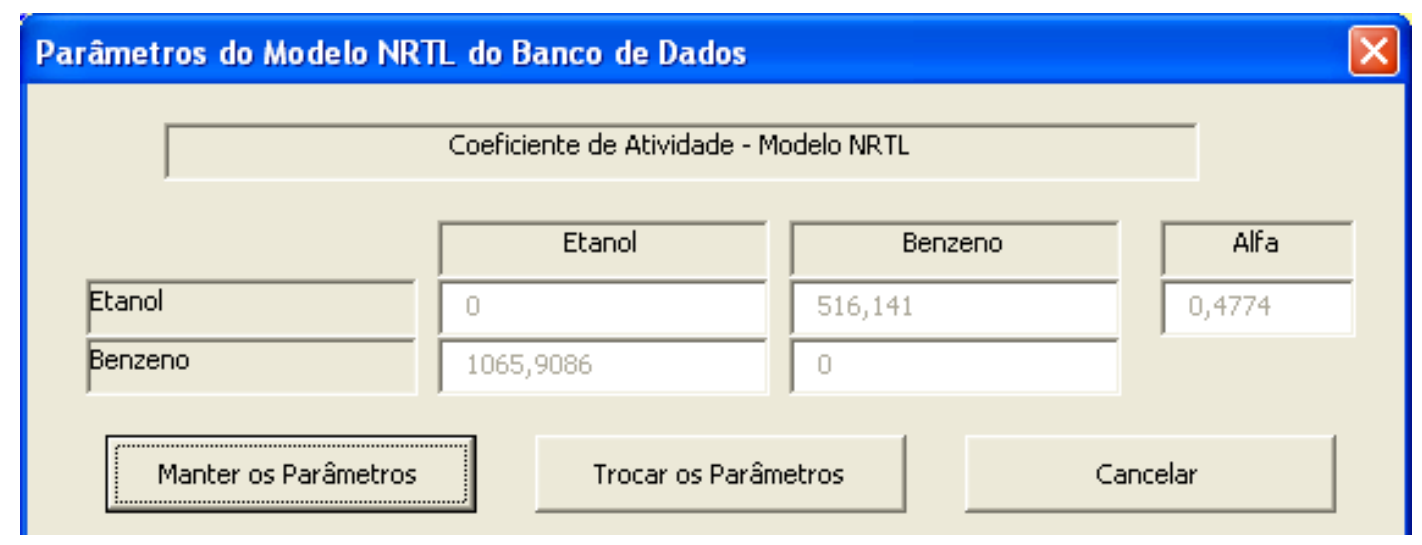

Os coeficientes de atividade utilizados no aplicativo para a correção da fase líquida são apresentados na Tabela 1. 
Tabela 1 - Modelos para a correção da fase líquida

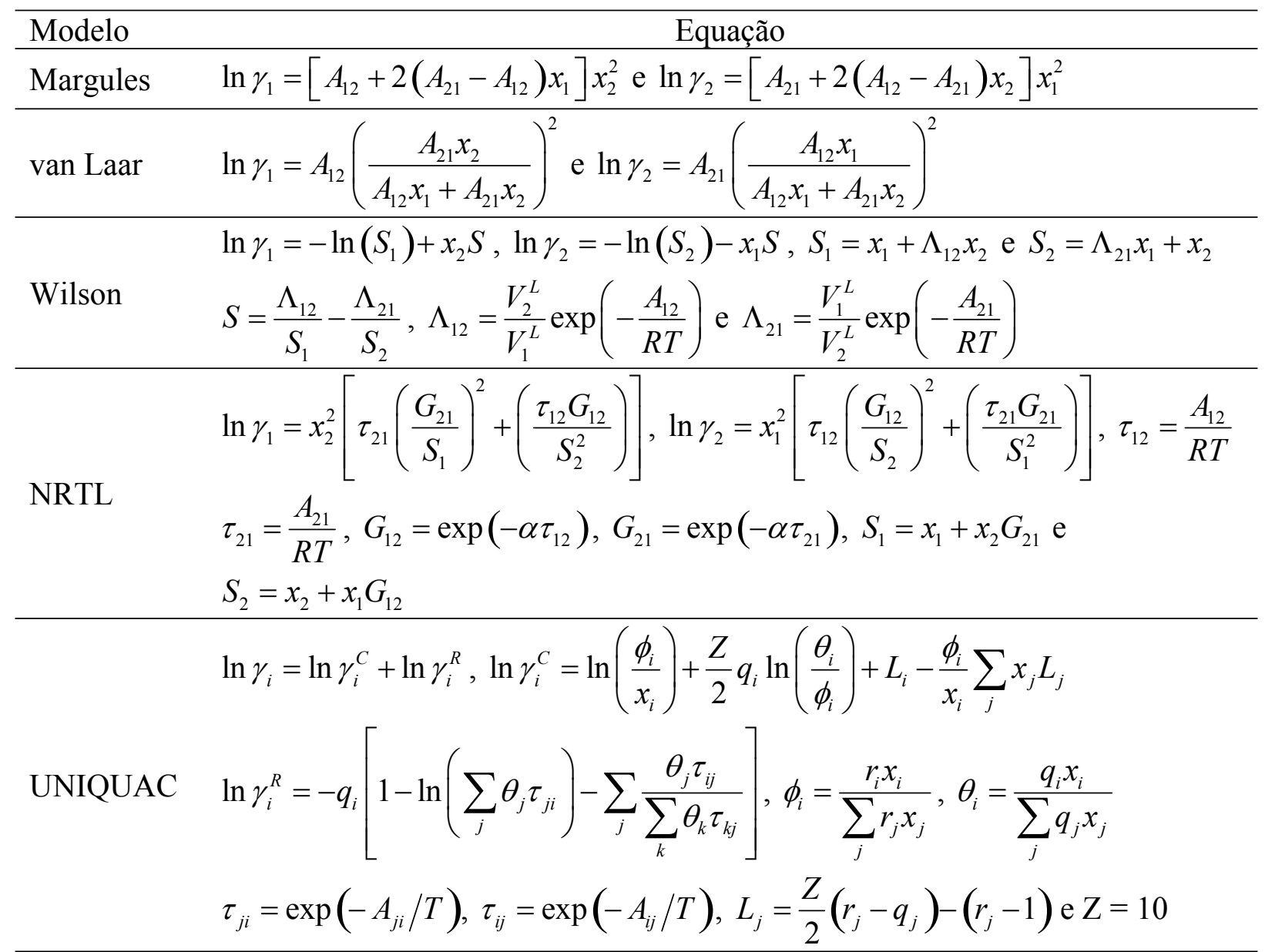

Constante universal dos gases: $R=1,98721 \mathrm{cal} / \mathrm{mol} . \mathrm{K}$

Escolha do tipo de cálculo: As opções disponíveis são apresentadas na Figura 6. Se a escolha do usuário for Um Ponto da Curva de Equilíbrio, os cálculos serão realizados através de um dos quatro problemas apresentados na Figura 7. Caso, contrário, a Curva de equilíbrio e o diagrama TXY são obtidos por meio do problema Bolha T (pressão do sistema disponível) ou Bolha P (temperatura do sistema disponível). Esta escolha é realizada na caixa de diálogo apresentada na Figura 8.

Figura 6- Cálculos disponíveis no aplicativo

\begin{tabular}{l} 
Escolha do Tipo de Cálculo \\
\begin{tabular}{|c|}
\hline Qual o Tipo de Cálculo? \\
c Um Ponto da Curva de Equilibrio \\
C Curva de equilibrio \\
\hline OK \\
\hline
\end{tabular} \\
\hline
\end{tabular}




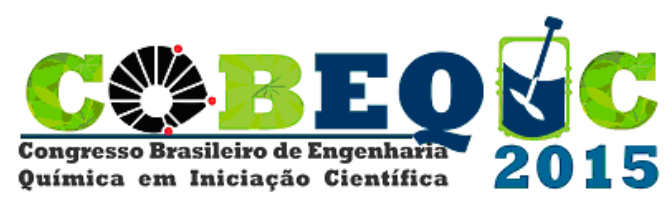

Figura 7 - Tipos de problemas ELV

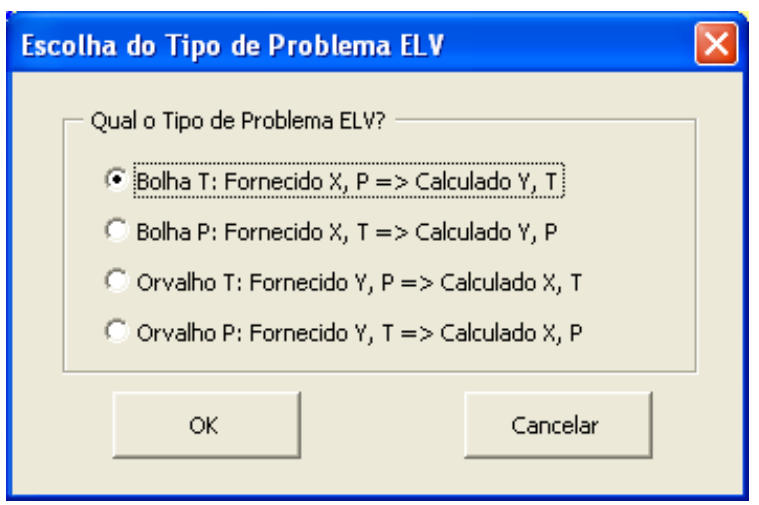

XI Congresso Brasileiro de Engenharia Química em Iniciação Científica Unicamp - Campinas - SP 19 a 22 de julho de 2015

Especificação do sistema: As especificações do sistema dependem do tipo de cálculo selecionado e do tipo de problema. As caixas de diálogos são mostradas nas Figuras 9 e 10:

Figura 9: Um ponto de equilíbrio

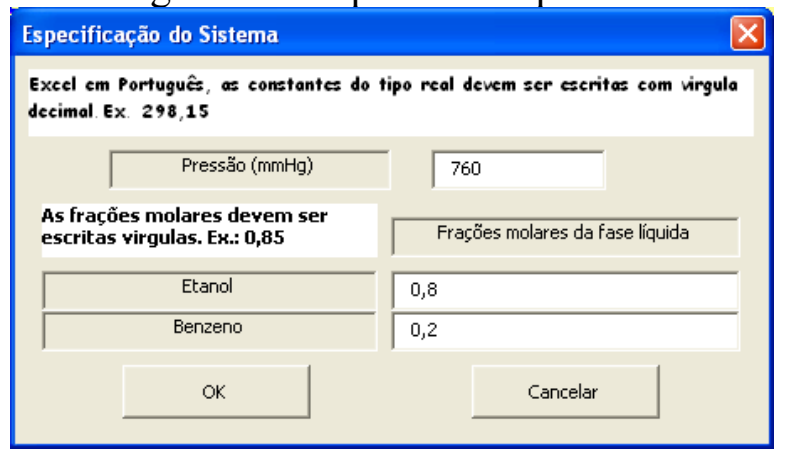

Figura 8 - Tipos de problemas ELV

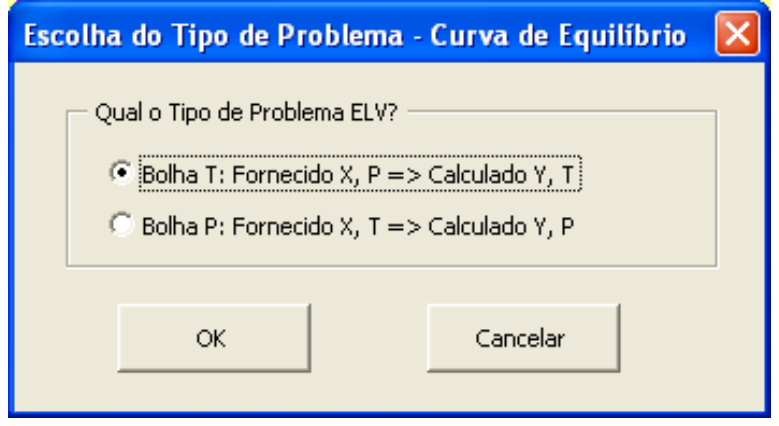

\section{RESULTADOS - VALIDAÇÃO DO APLICATIVO}

Vários sistemas binários reportados por Gmehling et al. (1982a, 1982b) foram utilizados para a validação do aplicativo proposto. Este trabalho descreve somente a comparação entre os valores das temperaturas de bolhas calculados pelo aplicativo com os reportadas por Gmehling et al. (1982a) para o sistema etanol(1)-benzeno(2) a $760 \mathrm{mmHg}$. A simbologia empregada é a seguinte: $\Delta T_{D e c}=T_{\exp }-T_{\text {calc }} \rightarrow$ DECHEMA e $\Delta T_{\text {Aplic }}=T_{\text {exp }}-T_{\text {calc }} \rightarrow$ Aplicativo. As diferenças e as temperaturas em ${ }^{\circ} \mathrm{C}$.

Tabela 2 - Diferenças entre as temperaturas de bolha experimentais e calculadas

\begin{tabular}{cc|cc|cc|cc|cc}
\hline \multicolumn{2}{c|}{ Margules } & \multicolumn{2}{c|}{ Van Laar } & \multicolumn{2}{c|}{ Wilson } & \multicolumn{2}{c}{ NRTL } & \multicolumn{2}{c}{ UNIQUAC } \\
\hline$\Delta \mathrm{T}_{\text {Dec }}$ & $\Delta \mathrm{T}_{\text {Aplic }}$ & $\Delta \mathrm{T}_{\text {Dec }}$ & $\Delta \mathrm{T}_{\text {Aplic }}$ & $\Delta \mathrm{T}_{\text {Dec }}$ & $\Delta \mathrm{T}_{\text {Aplic }}$ & $\Delta \mathrm{T}_{\text {Dec }}$ & $\Delta \mathrm{T}_{\text {Aplic }}$ & $\Delta \mathrm{T}_{\text {Dec }}$ & $\Delta \mathrm{T}_{\text {Aplic }}$ \\
\hline 0,02 & 0,01 & 0,05 & 0,04 & 0,11 & 0,60 & 0,03 & 0,50 & 0,05 & 0,37 \\
$-0,19$ & $-0,20$ & $-0,16$ & $-0,17$ & $-0,08$ & 0,68 & $-0,17$ & 0,57 & $-0,14$ & 0,39 \\
$-0,25$ & $-0,26$ & $-0,21$ & $-0,22$ & $-0,15$ & 0,70 & $-0,21$ & 0,60 & $-0,18$ & 0,42 \\
$-0,88$ & $-0,9$ & $-0,86$ & $-0,88$ & $-0,90$ & 0,01 & $-0,83$ & 0,01 & $-0,83$ & $-0,11$ \\
$-0,85$ & $-0,87$ & $-0,88$ & $-0,9$ & $-1,06$ & $-0,26$ & $-0,82$ & $-0,16$ & $-0,85$ & $-0,16$ \\
$-0,35$ & $-0,38$ & $-0,42$ & $-0,44$ & $-0,72$ & $-0,08$ & $-0,35$ & 0,04 & $-0,42$ & 0,16 \\
$-0,35$ & $-0,38$ & $-0,43$ & $-0,46$ & $-0,74$ & $-0,27$ & $-0,38$ & $-0,19$ & $-0,45$ & $-0,02$ \\
$-0,17$ & $-0,20$ & $-0,25$ & $-0,28$ & $-0,45$ & $-0,14$ & $-0,20$ & $-0,12$ & $-0,27$ & 0,04 \\
\hline
\end{tabular}


As diferenças verificadas na Tabela 2 são devido às constantes da equação de Antoine utilizada por Gmelhing et al. (1982a e 1982b) que diferem das utilizadas pelo aplicativo.

Nas Figuras 11 e 12 são mostradas as curvas de equilíbrio fornecidas pelo aplicativo dos sistemas acetona(1)-Clorofórmio(2) - desvios negativos em relação a lei de Raoult e etanol(1)-benzeno(2) - desvios positivos em relação a lei de Raoult.

Figura 11 - Sistema Acetona(1)-Clorofórmio(2) - Modelo NRTL
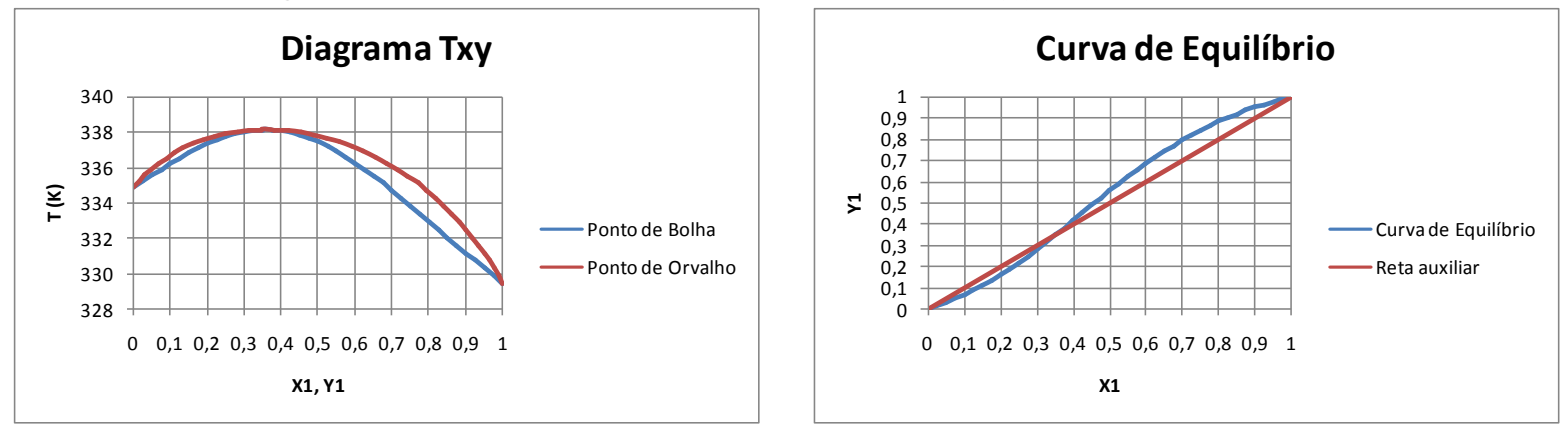

Figura 12 - Sistema Etanol(1)-Benzeno(2) - Modelo UNIQUAC
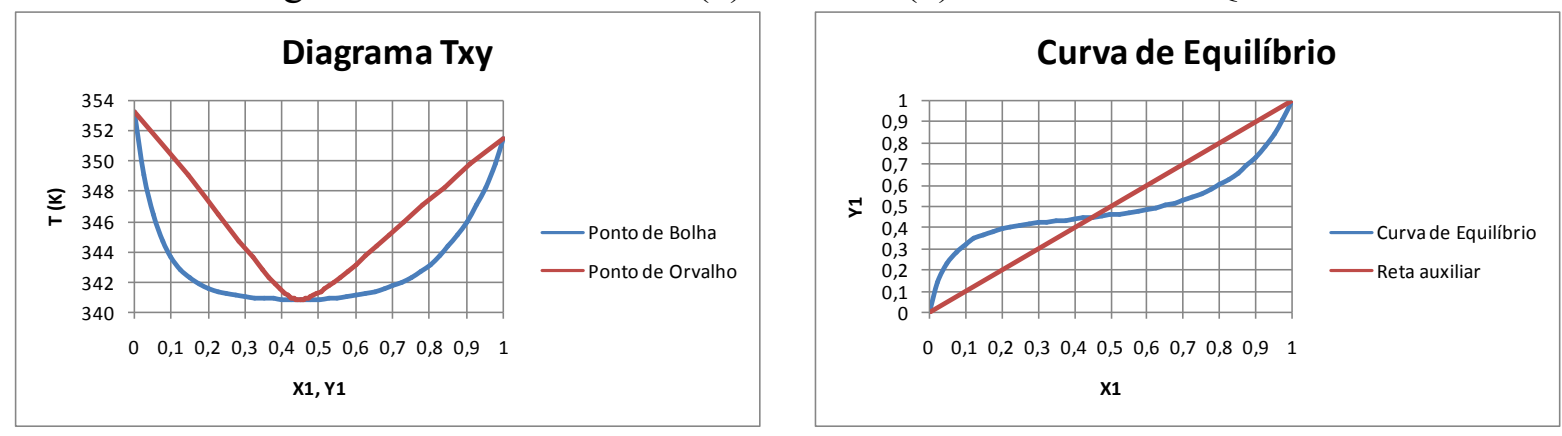

\section{CONCLUSÃO}

O aplicativo EXCEL/VBA desenvolvido mostrou-se eficiente para as estimativas de dados de equilíbrio líquido-vapor em pressões baixas. $\mathrm{O}$ aplicativo é interativo, o que torna fácil a sua utilização. Adicionalmente, o banco de dados do programa contém os parâmetros de interações binárias de 200 sistemas comumente empregados na indústria petroquímica.

\section{REFERÊNCIAS}

GMEHLING, J., ONKEN, U. WEIDLICH, U. Vapor-Liquid Equilibrium Data Collection, DECHEMA Chemistry Data Series. Vol. 1, DECHEMA 2C, 1982a.

GMEHLING, J., ONKEN, U. WEIDLICH, U. Vapor-Liquid Equilibrium Data Collection, DECHEMA Chemistry Data Series. Vol. 1, DECHEMA 2D, 1982 b.

KNUTH, D. E. Art of Computer Programming - V.3 - Sorting And Searching. Addison Wesley. Segunda Edição, 1998.

REID, R. C; PRAUSNITZ, J. M; SHERWOOD, T. K; The Properties of Gases and Liquids. McGraw-Hill, 1977 